\title{
Geometry on the variety of subspaces with positive definite form: geometry of the Grassmann spaces over generalized hyperbolic spaces
}

\author{
K. Prażmowski®i
}

\begin{abstract}
We consider Grassmann structures defined on the family consisting of subspaces on which a given nondegenerate bilinear form defined on a real vector space is positive definite. One may call such structures Grassmann spaces over generalized hyperbolic spaces. We show that the underlying (generalized) hyperbolic space can be recovered in terms of its Grassmannian, and the underlying projective space (equipped with respective associated polarity) can be recovered in terms of the generalized hyperbolic space defined over it.
\end{abstract}

Mathematics Subject Classification. 51M10, 51A45, 51A50.

Keywords. Grassmann space, projective space, hyperbolic space, exterior hyperbolic space.

\section{Introduction}

The aim of the paper is to investigate foundational questions concerning languages adequate to characterize geometry of grassmannian-like structures associated with - roughly speaking - hyperbolic spaces. On one side, hyperbolic geometry is often defined as a restriction of projective geometry to interior points of a real quadric (projective sphere: the Klein Model). This approach can be easily generalized to geometry on a convex body (with sufficiently regular boundary). Grassmannians and related adjacencies associated with such 'hyperbolic spaces' were studied in [8], where also fundamental characterization theorems were proved. On other side, while a quadric is determined by a bilinear form $\xi$, interior point $x$ of this quadric is characterized by the requirement $\xi(x, x)>0$. Yet, the above statement is not strict and precise. First 
of all, the forms $\xi$ and $-\xi$ determine the same quadric $Q$ consisting of the points $x$ such that $\xi(x, x)=0$. So, are the hyperbolic points characterized by $\xi(x, x)>0$ or by $\xi(x, x)<0$ ? A traditional answer depends on the (standard) signature of $\xi$. Secondly, the classical hyperbolic geometry is associated with the form of the type

$$
\xi(x, y)=x_{0} y_{o}-\sum_{i=1}^{n} x_{i} y_{i}
$$

As we shall see, many results concerning primitive notions of this hyperbolic geometry remain provable for arbitrary nondegenerate form. Thirdly, when we consider geometry of a restriction of a projective space to 'inner' points we must decide what are lines of this restriction. Are they projective lines consisting entirely of 'inner' points - this approach gives trivial structure inside a projective sphere; or they are intersections of projective lines with the set of 'inner' points. Then outside a classical projective sphere we obtain two distinct structures ('exterior hyperbolic spaces' in [4]). Analogous decision must be made when we define Grassmann spaces (structures on subspaces) associated with 'inner' points.

So, our results presented in the paper can be briefly sketched as follows. Given a symmetric bilinear nondegenerate form $\xi$ in a finite dimensional real vector space $\mathbb{V}$ we consider the set $\mathcal{L}(\xi)$ of subspaces of $\mathbb{V}$ such that $\xi(x, x)>0$ for all non-zero $x$ on that a subspace. The $k$-dimensional elements of $\mathcal{L}(\xi)=\mathcal{L}$ are the points of grassmannian structures we consider: spaces of pencils $\mathbf{P}_{k}(\mathcal{L})$, Grassmannians $\mathbf{G}_{k}(\mathcal{L})$, and restrictions $\mathbf{P}_{k}^{\#}(\mathcal{L})$ of the projective space of pencils $\mathbf{P}_{k}(\mathbb{V})$ (the projective $k$-Grassmannian, cf [5]) to $\mathcal{L}$. Assume that the form $\xi$ is defined by $\xi(x, y)=\sum_{i=1}^{r} x_{i} y_{i}-\sum_{r+1}^{n} x_{i} y_{i}$. If $r=1$ then $\mathbf{P}_{1}^{\#}(\mathcal{L}(\xi))$ coincides with the hyperbolic space defined on a projective disc, and $\mathbf{P}_{1}(\mathcal{L}(-\xi))$ is the corresponding exterior hyperbolic space. We generalize the result of [4] to arbitrary form $\xi$ and we prove (cf. Theorem 1.3) that the surrounding projective space can be defined in terms of $\mathbf{P}_{1}^{\#}(\mathcal{L}(\xi))$ and in terms of $\mathbf{P}_{1}(\mathcal{L}(\xi))$ when $\mathbf{P}_{1}(\mathcal{L}(\xi))$ has lines (there are 2-dimensional subspaces in $\mathcal{L}(\xi)$ i.e. $r \geq 2$ ).

In the last Sect. 3 we prove that the Grassmann structures $\mathbf{P}_{k}^{\#}(\mathcal{L}(\xi)), \mathbf{P}_{k}(\mathcal{L}(\xi))$, and $\mathbf{G}_{k}(\mathcal{L}(\xi))$ are (if nontrivial resp.) mutually definable: Propositions 3.9 and 3.13. Finally, we prove that the hyperbolic (exterior hyperbolic) spaces can be defined in terms of each of these Grassmann structures: Theorem 1.4.

At the end let us make a remark on the terminology used in the paper. We frequently say that a structure $\mathfrak{N}_{1}$ can be defined in terms of an other $\mathfrak{N}_{2}$, or $\mathfrak{N}_{1}$ can be recovered within $\mathfrak{N}_{2}$ meaning that suitable first order formulas exist in the language of $\mathfrak{N}_{2}$ which define a structure isomorphic (in a specific 'natural' and 'elementary' way) to $\mathfrak{N}_{1}$. A detailed exposition of this approach that may be specifically misleading when $\mathfrak{N}_{2}$ is originally defined on subsets of the universe of $\mathfrak{N}_{1}$, can be found in [9, Section 1.2]. Nevertheless, the naive understanding sketched above should suffice here. In particular, our results 
yield that each automorphism of $\mathfrak{N}_{2}$ determines an automorphism of $\mathfrak{N}_{1}$ so, mutually definable structures have essentially the same automorphism group.

\section{Main definitions: real generalized hyperbolic spaces and associated Grassmannians and spaces of pencils}

Let $\mathbb{V}$ be an $n$-dimensional real vector space, $\theta$ be its zero vector, and $\xi$ be a symmetric nondegenerate bilinear form defined on $\mathbb{V}$. There is an integer $r$, $0 \leq r \leq n$, such that in a suitable coordinate system of $\mathbb{V}$ the form $\xi$ is defined by the condition

$$
\begin{aligned}
& \xi(x, y)=\sum_{i=1}^{r} x_{i} y_{i}-\sum_{i=r+1}^{n} x_{i} y_{i} \\
& \text { where } x=\left[x_{1}, \ldots, x_{n}\right], y=\left[y_{1}, \ldots, y_{n}\right] .
\end{aligned}
$$

The pair $(r, n-r)$ is called the index (or the signature) of $\xi$; the set $\{r, n-r\}$ determines the form $\xi$ up to a linear equivalence. We assume that $r \geq 1$.

We write $\operatorname{Sub}_{k}(\mathbb{V})$ for the set of $k$-dimensional subspaces of $\mathbb{V}$. The form $\xi$ determines the orthogonality relation $\perp=\perp_{\xi}$ defined on vectors of $\mathbb{V}$ :

$$
u \perp v \Longleftrightarrow \xi(u, v)=0 .
$$

Clearly, $\perp$ is symmetric. The orthogonality $\perp$, as usually, is extended to arbitrary sets $U_{1}, U_{2}$ of vectors of $\mathbb{V}$ :

$$
U_{1} \perp U_{2} \Longleftrightarrow \forall u_{1}, u_{2}\left[u_{1} \in U_{1} \wedge u_{2} \in U_{2} \Longrightarrow u_{1} \perp u_{2}\right] ;
$$

we write $U^{\perp}=\{u:\langle u\rangle \perp U\}$ for any set $U$ of vectors. The symbol $\langle Z\rangle$ stands for the subspace of $\mathbb{V}$ spanned by $Z$. If $\langle U\rangle \in \operatorname{Sub}_{d}(\mathbb{V})$ for some integer $d$ then $U^{\perp} \in \mathrm{Sub}_{n-d}(\mathbb{V})$.

Let $k$ be a positive integer. If $\mathcal{D} \subset \operatorname{Sub}(\mathbb{V})=\bigcup_{d=0}^{n} \operatorname{Sub}_{d}(\mathbb{V})$ then we define $\mathcal{D}_{k}:=\mathcal{D} \cap \operatorname{Sub}_{k}(\mathbb{V})$. Given $H \in \mathcal{D}_{k-1}, H \subset B \in \mathcal{D}_{k+1}$ we define the $\mathcal{D}$-pencil with vertex $H$ and base $B$ :

$$
\mathbf{p}_{\mathcal{D}}(H, B):=\left\{U \in \mathcal{D}_{k}: H \subset U \subset B\right\} .
$$

With each such $\mathcal{D}$ and an integer $k$ we associate two 'Grassmannian-like' incidence structures (cf. e.g. [5], or incidence sequence of [2] or shadow space of $[3])$ :

$\mathbf{G}_{k}(\mathcal{D}):=\left\langle\mathcal{D}_{k}, \mathcal{D}_{k+1}, \subset\right\rangle$ - the $k$-th Grassmannian over $\mathcal{D}$, and $\mathbf{P}_{k}(\mathcal{D}):=\left\langle\mathcal{D}_{k}, \mathcal{P}_{k}(\mathcal{D}), \in\right\rangle$, where $\mathcal{P}_{k}(\mathcal{D})=\left\{\mathbf{p}(H, B): H \in \mathcal{D}_{k-1}, H \subset B \in\right.$ $\left.\mathcal{D}_{k+1}\right\}$ - the space of $k$-pencils over $\mathcal{D}$.

Clearly, in such a general setting, the structures $\mathbf{G}_{k}(\mathcal{D})$ and $\mathbf{P}_{k}(\mathcal{D})$ may degenerate/state trivial. 
The most important of them is the real $(n-1)$-dimensional projective space $\mathfrak{P}=\mathbf{P}_{1}(\operatorname{Sub}(\mathbb{V})) \cong \mathbf{G}_{1}(\operatorname{Sub}(\mathbb{V}))$. For short we write

$$
\mathbf{P}_{k}(\mathbb{V})=\mathbf{P}_{k}(\operatorname{Sub}(\mathbb{V})), \mathbf{G}_{k}(\mathbb{V})=\mathbf{G}_{k}(\operatorname{Sub}(\mathbb{V})) \text {, and } \mathbf{p}_{\operatorname{Sub}(\mathbb{V})}(H, B)=\mathbf{p}(H, B) .
$$

In most of the examples studied in our paper the considered family $\mathcal{D}$ is a lower set in the lattice $\langle\operatorname{Sub}(\mathbb{V}), \subset\rangle$, so $\mathbf{p}_{\mathcal{D}}(H, B)=\mathbf{p}(H, B)$ for any $B \in \mathcal{D}_{k+1}$.

Finally, for an arbitrary incidence structure $\mathfrak{M}=\langle S, \mathcal{L}\rangle(\mathcal{L} \subset \wp(S))$ and $X \subset S$ we define

$$
\mathcal{L} \mid X:=\{K \cap X: K \in \mathcal{L},|K \cap X| \geq 2\}, \quad \mathfrak{M} \uparrow X:=\langle X, \mathcal{L} \mid X\rangle ;
$$

$\mathfrak{M} \uparrow X$ is a partial linear space (a PLS), whenever $\mathfrak{M}$ is a PLS. We call $\mathfrak{M} \uparrow X$ the restriction of $\mathfrak{M}$ to $X$. If $\mathfrak{M}$ and $X$ are fixed in current investigations and $L$ is a line of $\mathfrak{M} \uparrow X$, then we write $\bar{L}$ for the line of $\mathfrak{M}$ such that $L \subset \bar{L}$.

In particular, for $\mathcal{D} \subset \operatorname{Sub}(\mathbb{V})$ we define

$$
\mathbf{P}_{k}^{\#}(\mathcal{D}):=\mathbf{P}_{k}(\mathbb{V}) \uparrow \mathcal{D}_{k} \text { - the projective space of } k \text {-pencils over } \mathcal{D} \text {. }
$$

With such a class $\mathcal{D}$ we associate its index ind:

$$
\operatorname{ind}(\mathcal{D}):=\max \left\{k: \mathcal{D}_{k} \neq \emptyset\right\} \text {. }
$$

The form $\xi$ defined on $\mathbb{V}$ gives rise to two important lower classes:

$$
\begin{aligned}
\mathcal{O}(\xi):=\{U \in \operatorname{Sub}(\mathbb{V}): \forall x \in U[\xi(x, x)=0]\} & \text { - the quadric determined } \\
& \text { by } \xi, \text { and } \\
\mathcal{L}(\xi):=\{U \in \operatorname{Sub}(\mathbb{V}): \forall x \in U[\xi(x, x)>0 \vee x=\theta]\} & \text { - the family of subspaces } \\
& \text { on which } \xi \text { is positive def- } \\
& \text { inite. }
\end{aligned}
$$

Both two classes determine their indexes; if $\xi$ is defined by (1) then

$$
\operatorname{ind}(\mathcal{O}(\xi))=\min \{r, n-r\} \quad \text { and } \quad \operatorname{ind}(\mathcal{L}(\xi))=r .
$$

Note that if $r=n$ then $\mathcal{L}(\xi)=\operatorname{Sub}(\mathbb{V})$ so, $\mathbf{P}_{k}(\mathcal{L}(\xi))=\mathbf{P}_{k}(\mathbb{V})$ for every integer $k$. To skip over this trivial case, finally, we assume that

$$
0<r<n \text {. }
$$

Clearly, $-\xi$ is also a nondegenerate symmetric bilinear form, its signature is $(n-r, r)$, and $\mathcal{O}(-\xi)=\mathcal{O}(\xi)$. One more property of the index of $\mathcal{D}=\mathcal{O}(\xi), \mathcal{L}(\xi)$ is widely used

$$
\begin{aligned}
& U \in \mathcal{D}, \operatorname{dim}(U)<\operatorname{ind}(\mathcal{D}) \Longrightarrow \exists D_{1}, D_{2} \in \mathcal{D}\left[D_{1} \neq D_{2} \wedge\right. \\
& \left.U \subset D_{1}, D_{2} \wedge \operatorname{dim}\left(D_{1}\right)=\operatorname{dim}\left(D_{2}\right)=\operatorname{dim}(U)+1\right] ;
\end{aligned}
$$

as a consequence of (4) we infer that after defining

$$
\mathcal{D}_{\text {max }}=\text { the set of elements of } \mathcal{D} \text { maximal under the inclusion }
$$


we have

$$
\mathcal{D}_{\max }=\mathcal{D}_{\operatorname{ind}(\mathcal{D})} .
$$

Note that the classes $\mathcal{O}(\xi)$ and $\mathcal{L}(\xi)$ satisfy the useful property

$$
\text { for each } U \in \operatorname{Sub}(\mathbb{V})\left[U \in \mathcal{D} \Longleftrightarrow \operatorname{Sub}_{1}(U) \subset \mathcal{D}_{1}\right] \text {. }
$$

But (6) is not a consequence of (4): consider any lower class $\mathcal{D}$ which satisfies (4). Let $1 \leq m<\operatorname{ind}(\mathcal{D})$ and define $\left.\mathcal{D}\right|_{m}=\{U \in \mathcal{D}: \operatorname{dim}(U) \leq m\}$. Then $\left.\mathcal{D}\right|_{m}$ is a lower class, it satisfies (4) but does not satisfy (6). Indeed, let $U \in \mathcal{D}_{\text {ind }(\mathcal{D})}$; then $\left.\operatorname{Sub}_{1}(U) \subset \mathcal{D}\right|_{m}$ but $\left.U \notin \mathcal{D}\right|_{m}$.

If a lower class $\mathcal{D}$ satisfies (4) then, clearly,

$$
\mathcal{D}_{k} \neq \emptyset \Longleftrightarrow k \leq \operatorname{ind}(\mathcal{D}) \text {, for any positive integer } k \text {. }
$$

Consequently, the following holds.

Fact 1.1. Let $\mathcal{D}=\mathcal{O}(\xi)$ or $\mathcal{D}=\mathcal{L}(\xi)$. Assume that $k \leq \operatorname{ind}(\mathcal{D})<n$. The structures $\mathbf{G}_{k-1}(\mathcal{D}), \mathbf{P}_{k-1}(\mathcal{D})$ and $\mathbf{P}_{k}^{\#}(\mathcal{D})$ are partial linear spaces.

The structure $\mathbf{Q}(\xi)=\mathbf{P}_{1}(\mathcal{O}(\xi)) \cong \mathbf{G}_{1}(\mathcal{O}(\xi))$ is the well known (real) polar space determined by $\xi$; it is a PLS whenever ind $(\mathcal{O}(\xi)) \geq 2$ and $\mathbf{P}_{k}(\mathcal{O}(\xi))$, denoted also by $\mathbf{P}_{k}(\xi)$ is the so called space of pencils of $(k-1)$ dimensional singular subspaces of $\mathbf{Q}(\xi)$, cf. $[2,6]$.

The structure $\mathbf{H}^{*}(\xi):=\mathbf{P}_{1}(\mathcal{L}(\xi))$ is a generalized exterior real hyperbolic space and then $\mathbf{H}(\xi):=\mathbf{P}(\mathbb{V}) \uparrow \mathcal{L}(\xi)=\mathbf{P}_{1}^{\#}(\mathcal{L}(\xi))$ is a generalized real hyperbolic space. To make an idea why we propose the names as above (and to compare it with commonly accepted terms) note that, in view of (1) for $x=\left[x_{1}, \ldots, x_{n}\right] \in$ V

$$
\langle x\rangle \in \mathcal{L}(\xi) \Longleftrightarrow x_{1}^{2}+\ldots+x_{r}^{2}>x_{r+1}^{2}+\ldots+x_{n}^{2} .
$$

So, indeed, if $r=1$ then $\mathcal{L}(\xi)_{1}$ consists of the points inside the projective sphere $S$ with equation $x_{1}^{2}=\sum_{i=2}^{n} x_{i}^{2}$ and $\mathbf{H}(\xi)$ is the Klein Model of the $(n-1)$ dimensional hyperbolic geometry, while if $r=n-1$ then $\mathcal{L}(\xi)_{1}$ consists of the points outside the sphere with equation $\sum_{i=1}^{n-1} x_{i}^{2}=x_{n}^{2} . \mathbf{H}(\xi)$ and $\mathbf{H}(-\xi)$ are linear spaces, but $\mathbf{H}^{*}(-\xi)$ is a (properly) partial linear space. Let us recall here one of the most fundamental known results.

Proposition 1.2. (cf. $[1,4])$ Let $n \geq 3$ and $\operatorname{ind}(\mathcal{L}(\xi))=1$. Then the projective space $\mathfrak{P}$ can be defined in terms of $\mathbf{H}(\xi)$ and $\mathbf{H}^{*}(-\xi)$.

Note. Let us stress here that for arbitrary form $\xi$ the structures $\mathbf{H}(\xi)$ and $\mathbf{H}^{*}(\xi)$ are not (necessarily) dual each to other; in essence their geometries are 'of dual type'. More detailedly, the dual of $\mathbf{H}(\xi)$ is $\mathbf{H}^{*}(-\xi)$, but this duality will not be exploited in the paper.

We shall generalize 1.2 to arbitrary (sensible) $n$ and $r$ : we prove our first main theorem. 
Theorem 1.3. Let $n \geq 3$ and $r=\operatorname{ind}(\mathcal{L}(\xi))$. The projective space $\mathfrak{P}$ equipped with the orthogonality $\perp_{\xi}$ can be defined in terms of

(i) $\mathbf{H}(\xi)$ when $r \geq 1$, and

(ii) $\mathbf{H}^{*}(\xi)$ when $r>1$.

Next, we pass to general situation and we investigate $\mathbf{P}_{k}(\mathcal{L}(\xi))$ and $\mathbf{G}_{k}(\mathcal{L}(\xi))$ for arbitrary integer $k$ such that $3 \leq k+1 \leq \operatorname{ind}(\mathcal{L}(\xi))$ and $\mathbf{P}_{k}^{\#}(\mathcal{L}(\xi))$ with $2 \leq k \leq \operatorname{ind}(\mathcal{L}(\xi))$. Note that if $U \in \mathcal{L}(\xi)$ then $\operatorname{Sub}_{1}(U) \cap \mathcal{O}(\xi)=\emptyset$ and therefore $U$ is regular wrt. $\xi$ i.e. $U \cap U^{\perp_{\xi}}=\{\theta\}$. So, the Grassmann structures investigated here contain corresponding Grassmannians of regular subspaces (cf. [10]). However, results concerning regular subspaces cannot be used here and our results are merely analogous to results of [10] and do not generalize them.

As in many geometries determined by a lower class $\mathcal{D}$ for a given $W \in \operatorname{Sub}_{d}(\mathbb{V})$ we define

$$
\begin{aligned}
& \text { the } \mathcal{D} \text {-star of } W=S_{\mathcal{D}}(W)=\left\{U \in \mathcal{D}_{d+1}: W \subset U\right\} \\
& \text { the } \mathcal{D} \text {-top of } W=T_{\mathcal{D}}(W)=\left\{U \in \mathcal{D}_{d-1}: U \subset W\right\} \text {. }
\end{aligned}
$$

It is evident that if $W \in \mathcal{D}$ then $T_{\mathcal{D}}(W)=T_{\mathrm{Sub}(\mathbb{V})}(W)=: T(W)$, while in general $S_{\mathcal{D}}(W) \subsetneq S_{\mathrm{Sub}(\mathbb{V})}(W)=: S(W)$. Let us write

$$
\begin{gathered}
\mathcal{S}_{k}(\mathcal{D}):=\left\{S_{\mathcal{D}}(H): H \in \mathcal{D}_{k-1}\right\}, \mathcal{T}_{k}(\mathcal{D}):=\left\{T_{\mathcal{D}}(B): B \in \mathcal{D}_{k+1}\right\}, \text { and } \\
\mathcal{T}_{k}^{*}(\mathcal{D}):=\left\{T_{\mathcal{D}}(B): B \in \operatorname{Sub}_{k+1}(\mathbb{V})\right\} .
\end{gathered}
$$

In the forthcoming 3.2 and 3.3 we characterize geometries induced by $\mathbf{P}_{k}(\mathcal{L}(\xi))$ and $\mathbf{P}_{k}^{\#}(\mathcal{L}(\xi))$ on the elements of $\mathcal{S}_{k}(\mathcal{L}(\xi)), \mathcal{T}_{k}(\mathcal{L}(\xi))$, and $\mathcal{T}_{k}^{*}(\mathcal{L}(\xi))$. These results enable us to use results concerning primitive notions of (generalized) hyperbolic geometry to study the structure of corresponding Grassmannianlike structures. In particular, we are able to reduce dimensions and transform investigations on $k$-th 'Grassmannians' into (equivalent) investigations on $(k-$ $1)$-th 'Grassmannians'. We close our investigations with the following theorem

Theorem 1.4. Let one of the following hold, where $\xi$ is defined by (1):

(1) $\mathfrak{M}=\mathbf{P}_{k}^{\#}(\mathcal{L}(\xi))$

(2) $\mathfrak{M}=\mathbf{P}_{k}(\mathcal{L}(\xi))$,

(3) $\mathfrak{M}=\mathbf{G}_{k}(\mathcal{L}(\xi))$.

Then $\mathbf{H}(\xi)$ can be defined in terms of $\mathfrak{M}$.

\section{On hyperbolic spaces}

Let us begin with recalling a one of classical techniques used to redefine projective plane $\mathbf{P}(\mathbb{V})$ which contains a given hyperbolic plane $\mathbf{H}(\xi)(r=1, n=3)$. 
The technique makes essential use of Desargues Configuration and Desargues Theorem. Let us write

$$
\left(\begin{array}{ccc|ccc}
a_{1} & b_{1} & c_{1} & C_{1} & A_{1} B_{1} \\
a_{2} & b_{2} & c_{2} & C_{2} & A_{2} B_{2} \\
a_{3} & b_{3} & c_{3} & C_{3} & A_{3} B_{3}
\end{array}\right)
$$

when $a_{i}, b_{i}, c_{i}, i=1,2,3$ are pairwise distinct points and $A_{i}, B_{i}, C_{i}, i=1,2,3$ are pairwise distinct lines (of a PLS whose incidence relation is denoted by $\|$ ) which satisfy the following incidences

$$
\begin{aligned}
a_{i}, a_{j}, c_{l} \| A_{l}, \quad b_{i}, b_{j}, c_{l} \mid B_{l} & \text { for all }\{i, j, l\}=\{1,2,3\}, \\
a_{i}, b_{i} \| C_{i} & \text { for all } i=1,2,3 .
\end{aligned}
$$

The classical Desargues theorem states the following:

Proposition 2.1. Let $a_{i}, b_{i}, c_{i}$ be pairwise distinct points and $A_{i}, B_{i}, C_{i}$ be pairwise distinct lines of a projective space coordinatized by a division ring. Assume that $\left(\begin{array}{lll|lll}a_{1} & b_{1} & c_{1} & C_{1} & A_{1} & B_{1} \\ a_{2} & b_{2} & c_{2} & C_{2} & A_{2} & B_{2} \\ a_{3} & b_{3} & c_{3} & C_{3} & A_{3} & B_{3}\end{array}\right)$ holds. The following conditions are equivalent

(i) there is a point o such that

$$
o \| C_{1}, C_{2}, C_{3}
$$

holds,

(ii) there is a line $O$ such that

$$
c_{1}, c_{2}, c_{3} \| O
$$

holds.

Let us fix a form $\xi$ and write, for short, $\mathfrak{H}=\mathbf{H}(\xi)$ and $\mathfrak{H}^{*}=\mathbf{H}^{*}(\xi)$. The following is crucial then

Proposition 2.2. Let $L_{1}, L_{2}, L_{3}$ be lines of the hyperbolic space $\mathfrak{H}$ and $M_{i}=\overline{L_{i}}$ be the corresponding lines of $\mathfrak{P}$ for $i=1,2,3$. The following conditions are equivalent.

(i) The lines $M_{1}, M_{2}, M_{3}$ are concurrent (in $\mathfrak{P}$ ).

(ii) The lines $L_{1}, L_{2}, L_{3}$ have a common point or there are pairwise distinct points $a_{i}, b_{i}, c_{i}$ and pairwise distinct lines $O, A_{i}, B_{i}$ of $\mathfrak{H}(i=1,2,3)$ such that $\left(\begin{array}{lll|lll}a_{1} & b_{1} & c_{1} & L_{1} & A_{1} & B_{1} \\ a_{2} & b_{2} & c_{2} & L_{2} & A_{2} & B_{2} \\ a_{3} & b_{3} & c_{3} & L_{3} & A_{3} & B_{3}\end{array}\right)$ holds in $\mathfrak{H}$ and $c_{1}, c_{2}, c_{3} \| O$.

Proof. In view of 2.1 the implication (ii) $\Longrightarrow$ (i) is evident. The implication (i) $\Longrightarrow$ (ii) is evident for $r=1\left(\mathcal{L}(\xi)_{1}\right.$ is a convex disc) but one can observe that it remains true for arbitrary index. Assume (i) and consider the least subspace $W$ of $\mathbb{V}$ such that $L_{1}, L_{2}, L_{2} \subset \operatorname{Sub}_{1}(W)$, then $3 \leq \operatorname{dim}(W) \leq 4$. The 
form $\xi \uparrow W$ may be degenerate but still we can apply definitions of Sect. 1 to construct the hyperbolic space $\mathbf{H}(\xi \uparrow W)$.

Let $q$ be the common point of $M_{1}, M_{2}, M_{3}$ and assume that $q \notin \mathcal{L}(\xi)$. Note that if $\operatorname{dim}(W)=4$, the lines $M_{1}, M_{2}, M_{3}$ are not coplanar in $\mathfrak{P}$.

In suitable coordinate system the form $\xi_{0}=\xi \uparrow W$ is defined by the formula

$$
\xi_{0}\left(\left[x_{1}, \ldots, x_{s}\right],\left[y_{1}, \ldots, y_{s}\right]\right)=\sum_{i=1}^{t} x_{i} y_{i}-\sum_{i=t+1}^{m} x_{i} y_{i} \text { with } t>0
$$

where $s=\operatorname{dim}(W)$. Note that if $s=m$ then $\xi_{0}$ is nondegenerate. Generally, the radical $R$ of $\xi_{0}$ is characterized by $x_{1}=\ldots=x_{m}=0$ so, $\operatorname{dim}(R)=s-m$. Writing $\left.x\right|_{m}=\left[x_{1}, \ldots, x_{m}\right]$ we can state the following

Fact 2.3. Let $x \in W . \xi(x, x)=\xi_{0}\left(\left.x\right|_{m},\left.x\right|_{m}\right)$. Then $x \in R$ implies $\langle x\rangle \notin \mathcal{L}(\xi)_{1}$. Suppose that $x \notin R$; then $\langle x\rangle \in \mathcal{L}(\xi)_{1}$ iff $\langle x+R\rangle \in \mathcal{L}(\xi / R)_{s-m+1}$.

In particular, if $t=m$ then $\xi_{0}(x, x)$ has constant sign outside $R$ and therefore $\mathbf{H}\left(\xi_{0}\right)$ is the slit space obtained by deleting from $\mathbf{P}(W)$ the 'hole' $R$. It is known that in this case a required system of points and lines exists (cf. [12]). So, when $\xi_{0}$ is degenerate it only remains to consider the following cases:

(a) $s=3, m=2, t=1\left(\right.$ so, $\left.\xi(x, y)=x_{1} y_{1}-x_{2} y_{2}\right)$;

(b) $s=4, m=2, t=1\left(\right.$ so, $\left.\xi(x, y)=x_{1} y_{1}-x_{2} y_{2}\right)$;

(c) $s=4, m=3, t=1\left(\operatorname{so}, \xi(x, y)=x_{1} y_{1}-x_{2} y_{2}-x_{3} y_{3}\right)$;

(d) $s=4, m=3, t=2\left(\operatorname{so}, \xi(x, y)=x_{1} y_{1}+x_{2} y_{2}-x_{3} y_{3}\right)$.

In case $(\mathrm{a}), \mathcal{O}\left(\xi_{0}\right)$ is a pair of lines in a real projective plane, and in case (b), $\mathcal{O}\left(\xi_{0}\right)$ is a pair of planes in a real projective 3-space. In corresponding cases the structure $\mathbf{H}\left(\xi_{0}\right)$ is a degenerate hyperbolic space (cf. [7]) i.e. a strip / a wall; it is a convex set in the surrounding projective space and it is seen that the required points and lines exist in $\mathbf{H}\left(\xi_{0}\right)$.

In cases $(\mathrm{c})$ and $(\mathrm{d})$ the set $\mathcal{O}\left(\xi_{0}\right)$ is a projective cone (a cylinder) and the points of $\mathbf{H}\left(\xi_{0}\right)$ are the points inside (outside, resp.) this cone. In case (c) the universe of $\mathbf{H}\left(\xi_{0}\right)$ is convex and the claim is also evident. By a side note observe that $\mathcal{L}\left(\xi_{0}\right)_{2}=\emptyset$ in the three above cases. In case (d) one should distinguish two cases: $q$ is the vertex of $\mathcal{O}\left(\xi_{0}\right)$, or $q$ is a point inside $\mathcal{O}\left(\xi_{0}\right)$ or it belongs to 'proper points' of $\mathcal{O}\left(\xi_{0}\right)$. In both cases elementary reasonings prove the claim (consider, auxiliary, a plane section of $\mathcal{L}\left(\xi_{0}\right)$ and projections of the lines $M_{i}$ onto this plane.

At the end let us pay attention to the most 'regular' case when $\xi_{0}$ is nondegenerate of the signature $(t, s-t)$. Cases $t=1$ and $t=s$ are evident so, it remains to consider the following cases:

(e) $s=3, t=2$;

(f) $s=4, t=2$; and

(g) $s=4, t=3$. 
In cases (e) and $(\mathrm{g})$ the set $\mathcal{O}\left(\xi_{0}\right)$ is the projective sphere (of dimension 1 and 2, resp.) and $\mathbf{H}\left(\xi_{0}\right)$ consists of the exterior points of this sphere. In both cases $\mathbf{H}\left(-\xi_{0}\right)$ and $\mathcal{O}\left(\xi_{0}\right)$ are (projectively) homogeneous so, without loss of generality, we can assume that $\mathcal{O}\left(\xi_{0}\right)$ is a sphere in the real affine (2 or 3 dimensional) space $\mathfrak{A}$ and $q$ is the centre of $\mathcal{O}\left(\xi_{0}\right)$ or $q$ is a point on $\mathcal{O}\left(\xi_{0}\right)$. In both cases there is a hyperplane $D$ of $\mathfrak{A}$ such that $\mathcal{O}\left(\xi_{0}\right)$ lies on one side of $D$ and $D$ is not parallel to any of the $M_{i}$. Then a required system of points and lines can be found in the other side of $D$.

In case (f) the set $\mathcal{O}\left(\xi_{0}\right)$ is a ruled quadric in the real projective 3-space so, it divides this space into two regions and the set of points of $\mathbf{H}\left(\xi_{0}\right)$ is one of these two regions. $\mathbf{H}\left(-\xi_{0}\right)$ and $\mathcal{O}\left(\xi_{0}\right)$ are projectively homogeneous and, as above, it suffices to consider an 'affine part' of $\mathbf{H}\left(\xi_{0}\right)$, embedded into the real affine 3 -space such that $q=(0,0,0)$ and either $\mathcal{O}\left(\xi_{0}\right)$ is characterized by the equation $x_{1}^{2}+x_{2}^{2}=x_{3}^{2}+1\left(q \notin \mathcal{O}\left(\xi_{0}\right)\right)$ or $\mathcal{O}\left(\xi_{0}\right)$ is defined by the equation $x_{1}^{2}-x_{2}^{2}=x_{3}\left(q \in \mathcal{O}\left(\xi_{0}\right)\right)$. In the first case $\mathcal{O}\left(\xi_{0}\right)$ (restricted to $\left.\mathfrak{A}\right)$ is a one sheet hyperboloid and in the second case it is a hyperbolic paraboloid.

Let $q \notin \mathcal{O}\left(\xi_{0}\right)$. For every $i=1,2,3$ we consider an open halfline $N_{i}$ with origin $q$ contained in $M_{i}$; we can choose $N_{i}$ such that $N_{i} \cap \mathcal{L}\left(-\xi_{0}\right)_{1}$ is a segment and $N_{i} \cap \mathcal{L}\left(\xi_{0}\right)$ is a halfline. For all $1 \leq i<j \leq 3$ there is a line of $\mathfrak{P}$ entirely contained in $\mathcal{L}\left(\xi_{0}\right)_{1}$ which crosses $N_{i}, N_{j}$. Finally, we note that a triangle with the vertices on the $N_{i}$ and the sides entirely contained in $\mathcal{L}\left(\xi_{0}\right)_{1}$ exists. Then the claim of (ii) becomes evident.

Let $q \in \mathcal{O}\left(\xi_{0}\right)$; now our reasoning becomes more complex. Note that at most two of the $M_{i}$ are tangent to $\mathcal{O}\left(\xi_{0}\right)$. Assume, first, that $M_{1}, M_{2}$ are tangent so, the equation of $M_{i}$ is $x_{2}=\alpha_{i} x_{1}, x_{3}=0(i=1,2)$ with $\alpha_{1}^{2}, \alpha_{2}^{2}>1$. Without loss of generality we can assume that $M_{3}$ is the $x_{3}$-axis. There is a triangle with the vertices $a_{1}, a_{2}, a_{3}$ inscribed into the $M_{i}\left(a_{i} \in M_{i}\right)$ such that the joining lines $\overline{a_{1}, a_{3}}, \overline{a_{2}, a_{3}}$ are contained in $\mathcal{L}\left(\xi_{0}\right)_{1}$. As a second triangle $b_{1}, b_{2}, b_{3}$ we can choose a one parallel to $a_{1}, a_{2}, a_{3}$.

Next, we suppose that $M_{1}$ is tangent to $\mathcal{O}\left(\xi_{0}\right)$, and $M_{2}, M_{3}$ are not tangent. Now, we can assume that $M_{3}$ is the $x_{3}$-axis, $M_{1}$ is characterized by $x_{2}=$ $\alpha x_{1}, x_{3}=0, \alpha>1$, and $M_{2}$ has equation of the form $x_{3}=\beta x_{2}, x_{1}=0$. Computations similar to the above, though more complicated, prove our claim.

Finally, we assume that no one of the $M_{i}$ is tangent to $\mathcal{O}\left(\xi_{0}\right)$. In this case we change again the coordinate system so as $\mathcal{O}\left(\xi_{0}\right)$ becomes a hyperbolic paraboloid (with the equation as before) and $q$ is the direction of the $x_{3}$-axis. Now the claim is evident - consider (auxiliary) a plane of $\mathfrak{A}$ parallel to the $x_{1} x_{2}$-plane which crosses each of the $M_{i}$ in a point in $\mathcal{L}\left(\xi_{0}\right)$.

Note. As we read from the proof of 2.2 it may be a hard task to find required triangles $a_{i}, b_{i}$ for given concurrent lines $\overline{L_{1}}, \overline{L_{2}}, \overline{L_{3}}$. But a formula which defines the concurrency is relatively simple. One can also follow another way: suppose that (ii) of 2.2 defines some relation $\boldsymbol{\rho}^{\prime}$ between lines $L_{1}, L_{2}, L_{3}$. It is 
simpler to prove now $\overline{L_{1}}, \overline{L_{2}}, \overline{L_{3}}$ are concurrent in $\mathfrak{P}$ iff there are distinct lines $L^{\prime}, L^{\prime \prime}$ such that $\boldsymbol{\rho}^{\prime}\left(L^{\prime}, L^{\prime \prime}, L_{i}\right)$ holds for $i=1,2,3$.

With a slight abuse of language we say that lines $L_{1}, L_{2}, L_{3}$ of $\mathfrak{H}$ are concurrent in $\mathfrak{P}$ and we write $\boldsymbol{\rho}\left(L_{1}, L_{2}, L_{3}\right)$ when $\overline{L_{1}}, \overline{L_{2}}, \overline{L_{3}}$ are concurrent in $\mathfrak{P}$. In view of 2.2 , the relation $\rho$ is definable in $\mathfrak{H}$ so, the points of $\mathfrak{P}$ can be defined in $\mathfrak{H}$ as equivalence classes $\left[L_{1}, L_{2}\right]_{\rho}$ of distinct lines $L_{1}, L_{2}$ of $\mathfrak{H}$ - one sees that through every point $p$ of $\mathfrak{P}$ there pass at least two lines $L_{1}, L_{2}$ of $\mathfrak{H}$ such that $p \in \overline{L_{1}}, \overline{L_{2}}$.

Let $p_{1}, p_{2}$ be points of $\mathfrak{P}$ not in $\mathfrak{H}, p_{i}$ be the equivalence class of a pair $\left(L_{i}^{\prime}, L_{i}^{\prime \prime}\right)$, and $a_{1}, a_{2}$ be points of $\mathfrak{H}$. The following is evident and trivial.

- $a_{1}, a_{2}, p_{1}$ are collinear in $\mathfrak{P}$ iff there is a line $L$ of $\mathfrak{H}$ such that $a_{1}, a_{2} \in L$, $\boldsymbol{\rho}\left(L, L_{1}^{\prime}, L_{1}^{\prime \prime}\right)$,

- $a_{1}, p_{1}, p_{2}$ are collinear in $\mathfrak{P}$ iff there is a line $L$ of $\mathfrak{H}$ such that $a_{1} \in L$ and $\boldsymbol{\rho}\left(L, L_{i}^{\prime}, L_{i}^{\prime \prime}\right)$ for $i=1,2$.

Finally, we note the following lemma, which enables us to (re)define the collinearity relation on $\mathfrak{P}$.

Lemma 2.4. Let $p_{i}=\overline{L_{i}^{\prime}} \cap \overline{L_{i}^{\prime \prime}}$ for $i=1,2,3$ be pairwise distinct points of $\mathfrak{P}$ not in $\mathfrak{H}$, where $L_{i}^{\prime}, L_{i}^{\prime \prime}$ are lines of $\mathfrak{H}$. The following conditions are equivalent

(i) $p_{1}, p_{2}, p_{3}$ are collinear in $\mathfrak{P}$;

(ii) there are in $\mathfrak{H}$ pairwise distinct points $o, a_{i}, b_{i}$ and pairwise distinct lines

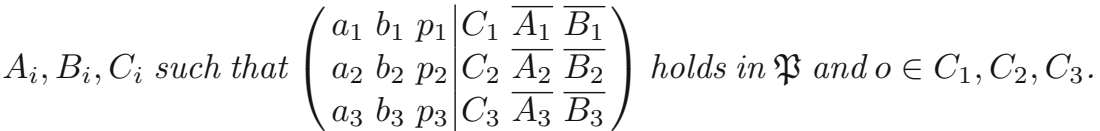

Note that the assumptions of (7) like $p_{i} \mid \overline{A_{i}}$ are expressible in $\mathfrak{H}$ by formula of the form $\rho\left(L_{i}^{\prime}, L_{i}^{\prime \prime}, A_{i}\right)$.

Proof. (ii) $\Longrightarrow$ (i) is a direct consequence of 2.1. Assume (i) and let $L_{0}$ be a line of $\mathfrak{P}$ through $p_{1}, p_{2}, p_{3}$. Let $o$ be a point of $\mathfrak{H}$, and $\Pi=\overline{L_{0} \cup\{o\}}$ be the plane of $\mathfrak{P}$ through $L_{0}, o$. Then $Q_{0}=\mathbf{Q}(\xi) \cap \Pi$ is either a conic or a pair of (isotropic) lines such that $Q_{0}$ separates $\mathcal{L}(\xi)_{1} \cap \Pi$ and $L_{0}$. It is easy to find in $\mathcal{L}(\xi)$ on $\Pi$ a required system of points and lines.

This proves that $\mathfrak{P}$ can be defined in terms of $\mathfrak{H}$. It is more difficult to define $\mathfrak{P}$ in terms of $\mathfrak{H}^{*}$, because $\mathfrak{H}^{*}$ is only partially linear - it has 'less' lines. In essence, our first step consists in defining $\mathfrak{H}$ in terms of $\mathfrak{H}^{*}$ ! Again, to this aim we use the Desargues Theorem.

Assume that $\operatorname{ind}(\mathcal{L}) \geq 2$ so, $\mathfrak{H}^{*}$ is nontrivial.

Lemma 2.5. Let $q_{1}, q_{2}, q_{3}$ be distinct points of $\mathfrak{H}^{*}$. The following conditions are equivalent:

(i) there is a line $L_{0}$ of $\mathfrak{H}$ through $q_{1}, q_{2}, q_{3}$; 
(ii) $q_{1}, q_{2}, q_{3}$ are on a line of $\mathfrak{H}^{*}$ or there are in $\mathfrak{H}^{*}$ pairwise distinct points $o, a_{i}, b_{i}$ and pairwise distinct lines $A_{i}, B_{i}, C_{i}$ such that the relation $\left(\begin{array}{lll|lll}a_{1} & b_{1} & q_{1} & C_{1} & A_{1} & B_{1} \\ a_{2} & b_{2} & q_{2} & C_{2} & A_{2} & B_{2} \\ a_{3} & b_{3} & q_{3} & C_{3} & A_{3} & B_{3}\end{array}\right)$ holds in $\mathfrak{P}$ (equivalently: in $\mathfrak{H}^{*}$ ) and $o \in C_{1}, C_{2}, C_{3}$.

Proof. In view of 2.1 only the (i) $\Longrightarrow$ (ii) implication needs a proof. Let $q_{1}, q_{2}, q_{3}$ lie on a line $L_{0}$ of $\mathfrak{P}$. If $L_{0} \subset \mathcal{L}(\xi)_{1}$ then $L_{0}$ is a line of $\mathfrak{H}^{*}$ so, we assume that $L_{0}$ contain points outside $\mathcal{L}(\xi)$. There is a line $L_{0}^{\prime}$ through $q_{1}$ entirely contained in $\mathcal{L}(\xi)_{1}$; let $\Pi$ be the plane of $\mathfrak{P}$ which contains $L_{0} \cup L_{0}^{\prime}$. Then $\Pi \cap \mathcal{O}(\xi)_{1}$ is a conic $\mathcal{C}$ on $\Pi, \mathcal{L}(\xi)_{1} \cap \Pi$ consists of the points outside $\mathcal{C}$ and $L_{0}$ is either a secant or a tangent of $\mathcal{C}$. Let $\mathfrak{A}$ be the affine plane obtained by deleting $L_{0}$ from $\Pi$. Without loss of generality in the corresponding cases we can assume that one of the following holds in a suitable coordinate system of $\mathfrak{A}$ :

- $\mathcal{C}$ is defined on $\mathfrak{A}$ by the equation $x \cdot y=1$, the points of $\mathcal{L}(\xi)$ on $\mathfrak{A}$ are characterized by $x \cdot y<1$, and an affine line with equation $y=\alpha x+\beta$ is in $\mathfrak{H}^{*}$ when $|\beta|<2 \sqrt{\alpha}$ (i.e. $\beta^{2}<4 \alpha$ )

- $\mathcal{C}$ is defined on $\mathfrak{A}$ by the equation $y=x^{2}$, the points of $\mathcal{L}(\xi)$ on $\mathfrak{A}$ are characterized by $y<x^{2}$, and an affine line with equation $y=\alpha x+\beta$ is in $\mathfrak{H}^{*}$ when $\beta<2 \alpha^{2}$.

In both cases the points $q_{1}, q_{2}, q_{3}$ are directions of lines of $\mathfrak{A}$ which are in $\mathfrak{H}^{*}$. It is relatively easy to compute directly coordinates of required points $a_{i}, b_{i}, o$.

Combining together 2.5, 2.4, and 2.2 we get that $\mathfrak{P}$ can be defined in terms of $\mathfrak{H}$ and $\mathfrak{H}^{*}$, respectively. To complete a proof of 1.3 we note the following fact.

Fact 2.6. The orthogonality $\perp_{\xi}$ can be defined in terms of the projective space $\mathfrak{P}$ with its subset $\mathcal{L}(\xi)_{1}$ distinguished.

Proof. Let us write, for short: $\mathcal{H}=\mathcal{L}(\xi)_{1}, \mathcal{Q}=\mathcal{O}(\xi)_{1}$, and $\mathcal{H}^{*}=\mathcal{L}(-\xi)_{1}$. Then the point set of $\mathfrak{P}$ is the disjoint union $\mathcal{H} \cup \mathcal{Q} \cup \mathcal{H}^{*}$. The proof of Fact 2.6 is provided in two steps.

First step. $\mathcal{Q}$ is definable in $(\mathfrak{P}, \mathcal{H})$.

For every line $L$ of $\mathfrak{P}$ which touches $\mathcal{Q}$ in a point $a$ one of the following holds

$$
\text { (a) } L \backslash\{a\} \subset \mathcal{H} \text { or } \quad \text { (b) } L \backslash\{a\} \subset \mathcal{H}^{*} \text {. }
$$

If $L$ and $\mathcal{Q}$ are not tangent then $|L \cap \mathcal{Q}| \in\{0,2\}$.

Clearly, either

(i) $r=1$ or $r=n-1$, or

(ii) $1<r<n-1$ 
In case (ii) for every point $a \in \mathcal{Q}$ there are lines $L_{1}, L_{2}$ through $a$ such that (a) holds for $L_{1}$ and (b) holds for $L_{2}$. Consequently, for a point $a$ of $\mathfrak{P}$ we have

$$
a \in \mathcal{Q} \Longleftrightarrow \text { there exists a line } L \text { of } \mathfrak{P} \text { s.t. } L \backslash\{a\} \subset \mathcal{H} \text {. }
$$

If $r=n-1$ then (a) holds for every line $L$ tangent to $\mathcal{Q}$ in $a$ and therefore (11) remains valid.

Assume that $r=1$; then $\mathcal{H}$ is the Klein Model inside the projective sphere $\mathcal{Q}$ and all tangent lines are exterior, so (b) holds for them. In this case, however, results concerning classical hyperbolic spaces can be used to justify our step. In essence. firstly, we define in the induced hyperbolic space the notion of a maximal triangle (also called an asymptotic triangle) and then the hyperbolic parallelism. The details of this way can be found in [11, Proposition 1.4 and corresponding figures], given for Strambach planes but valid for hyperbolic spaces as well.

Second step. The orthogonality $\perp_{\xi}$ can be defined in $(\mathfrak{P}, \mathcal{Q})$.

With $\mathcal{Q}$ distinguished the family $\mathcal{G}$ of lines of $\mathfrak{P}$ tangent to $\mathcal{Q}$ can be distinguished as well. The point is to define the correlation

$$
a \longmapsto a^{\perp \xi}=: \varkappa(a)
$$

in terms of $\mathfrak{P}$ and $\mathcal{Q}$.

Let $a \in \mathcal{Q}$; then $\varkappa(a)$ is the union of the lines tangent to $\mathcal{Q}$ at $a$.

Next, let $\mathcal{Q}^{\prime}$ consist of points $a$ of $\mathfrak{P}$ such that $a \notin \mathcal{Q}$ and there are lines in $\mathcal{G}$ through $a$. Then $\varkappa(a)$ is the hyperplane of $\mathfrak{P}$ spanned by all the points $b \in \mathcal{Q}$ such that $a \in \varkappa(b)$.

Finally, if there is a point $a$ such that all lines in $\mathcal{G}$ miss it ( $a$ is an interior point of the respective sphere) then $b \in \varkappa(a)$ if $b$ lies on a line which contains two points $b_{1}, b_{2} \in \mathcal{Q}^{\prime}$ such that $a \in \varkappa\left(b_{1}\right), \varkappa\left(b_{2}\right)$.

Now the proof of 2.6 is complete.

\section{Grassmann structures}

Let us begin with a charcterization of geometries on $\mathcal{L}(\xi)$-stars and $\mathcal{L}(\xi)$-tops. Let $H \in \mathcal{L}(\xi)_{k-1}$, then $H^{\perp}$ is regular so, $\xi \uparrow H^{\perp}$ is nondegenerate. One can easily note that if $(r, n-r)$ is the signature of $\xi$ (and therefore $k-1 \leq r)$ then the signature of $\xi \uparrow H^{\perp}$ equals to $(r-k+1, n-r)$. It is known that the map

$$
\operatorname{Sub}_{1}\left(H^{\perp}\right) \ni\langle u\rangle \longmapsto H+\langle u\rangle
$$

is a bijection between $\operatorname{Sub}_{1}\left(H^{\perp}\right)$ and $S(H)$; its inverse is the map $U \longmapsto$ $U \cap H^{\perp}$. It is rather easy to note the following fact. 
Fact 3.1. The map defined above transforms bijectively $\mathcal{L}\left(\xi \uparrow H^{\perp}\right)_{1}$ onto $S_{\mathcal{L}(\xi)}(H)$. Generally, the image of $\mathcal{L}\left(\xi\left\lceil H^{\perp}\right)_{m}\right.$ under this map is the set $\left\{\operatorname{Sub}_{k}(B): H \subset\right.$ $\left.B \in \mathcal{L}(\xi)_{k+m-1}\right\}$.

As an evident consequence we obtain then

Proposition 3.2. Let $B \in \mathcal{L}(\xi)_{k+1}, H \in \mathcal{L}(\xi)_{k-1}$.

(i) The restrictions to $T_{\mathcal{L}(\xi)}(B)$ of each of the following two: $\mathbf{P}_{k}(\mathcal{L}(\xi))$, $\mathbf{P}_{k}^{\#}(\mathcal{L}(\xi))$ is the projective space dual to $\mathbf{P}(B)$. Its (geometrical) dimension is $k$. Clearly, $T_{\mathcal{L}(\xi)}(B)$ is a line of $\mathbf{G}_{k}(\mathcal{L}(\xi))$.

(ii) The restrictions to $S_{\mathcal{L}(\xi)}(H)$ of $\mathbf{P}_{k}^{\#}(\mathcal{L}(\xi)), \mathbf{P}_{k}(\mathcal{L}(\xi))$, and $\mathbf{G}_{k}(\mathcal{L}(\xi))$ are (isomorphic to $) \mathbf{P}_{1}^{\#}\left(\mathcal{L}\left(\xi\left\lceil H^{\perp}\right)\right), \mathbf{P}_{1}\left(\mathcal{L}\left(\xi \uparrow H^{\perp}\right)\right)\right.$, and $\mathbf{G}_{1}\left(\mathcal{L}\left(\xi \uparrow H^{\perp}\right)\right)$ resp. Consequently, the first of them is the hyperbolic space $\mathbf{H}\left(\xi\left\lceil H^{\perp}\right)\right.$ and the two next are the exterior hyperbolic space $\mathbf{H}^{*}\left(\xi \uparrow H^{\perp}\right)$. Their geometrical dimension is $n-k$, and the (linear) index is $r-k+1$.

A similar fact will be also useful.

Proposition 3.3. Let $H \in \operatorname{Sub}_{k-1}(\mathbb{V}), B \in \operatorname{Sub}_{k+1}(\mathbb{V})$.

(i) If $H \notin \mathcal{L}(\xi)$ then $S_{\mathcal{L}(\xi)}(H)=\emptyset$.

(ii) If $T_{\mathcal{L}(\xi}(B) \neq \emptyset$ then $\operatorname{ind}(\xi \uparrow B) \in\{k, k+1\}$. The case when $\operatorname{ind}(\xi \uparrow$ $B)=k+1$ was considered in 3.2(i) so, let $\operatorname{ind}(\xi \uparrow B)=k$ and set $\mathcal{X}=T_{\mathcal{L}(\xi)}(B)$.

The restriction of $\mathbf{P}_{k}(\mathcal{L}(\xi))$ to $\mathcal{X}$ is a trivial structure: it has no lines. If $\xi_{0}:=\xi \uparrow B$ is nondegenerate then the correlation of $\mathbf{P}(B)$ determined by $\xi_{0}$ is an isomorphism of the restriction of $\mathbf{P}_{k}^{\#}(\mathcal{L}(\xi))$ to $\mathcal{X}$ and the hyperbolic space $\mathbf{H}\left(\xi_{0}\right)$, whose geometrical dimension is $k$ and whose form has index $k$ as well. So, it is the $k$-dimensional real hyperbolic space.

If $\xi_{0}$ is degenerate then the restriction of $\mathbf{P}_{k}^{\#}(\mathcal{L}(\xi))$ to $\mathcal{X}$ is a $k$-dimensional affine space.

Proof. (i) is evident. To justify (ii) note that the form $\xi_{0}$, if nondegenerate, in a suitable coordinate system has the form $\xi_{0}(x, y)=\sum_{i=1}^{k} x_{i} y_{i}-x_{k+1} y_{k+1}$. Then $\mathcal{O}\left(\xi_{0}\right)$ is a projective sphere. A hyperplane of $\mathbf{P}(B)$ belongs to $\mathcal{L}\left(\xi_{0}\right)$ iff it is exterior wrt. to this sphere and then the correlation in question maps the hyperplane onto a point inside $\mathcal{O}\left(\xi_{0}\right)$.

If $\xi_{0}$ is degenerate i.e. it has the form $\xi(x, y)=\sum_{i=1}^{k} x_{i} y_{i}$ then $\xi_{0}(x, x) \geq 0$ for all $x$ on $B$ and $\mathcal{O}\left(\xi_{0}\right)$ consists of the single point $[0, \ldots, 0,1]=: a$. Thus $\mathcal{L}\left(\xi_{0}\right)_{k}$ consists of the hyperplanes of $\mathbf{P}(B)$ which miss $a$. The geometry of this restriction is the affine one.

Next, we aim to define families of respective stars and tops in terms of the surrounding Grassmanian-like structures. 
Let $\Delta$ be a triangle in a partial linear space $\mathfrak{M}$, let $q_{1}, q_{2}, q_{3}$ be the sides of $\Delta$, $U_{1}, U_{2}, U_{3}$ be its vertices, and $U$ be a point of $\mathfrak{M}$. Consider the following two properties

$$
U \text { is collinear in } \mathfrak{M} \text { with the vertices } U_{1}, U_{2}, U_{3} \text { of } \Delta \text {; }
$$

$U$ is collinear in $\mathfrak{M}$ with the vertices of a triangle inscribed into $q_{1} \cup q_{2} \cup q_{3}$.

Let us recall the following classical result.

Fact 3.4. Let $\mathfrak{M}=\mathbf{P}_{k}(\mathbb{V})$ and let $\Delta$ be a triangle in $\mathfrak{M}$, as above. Then either

(a) $q_{1} \cup q_{2} \cup q_{3} \subset S(H)$ for an $H \in \operatorname{Sub}_{k-1}(\mathbb{V})$, or

(b) $q_{1} \cup q_{2} \cup q_{3} \subset T(B)$ for $a B \in \operatorname{Sub}_{k+1}(\mathbb{V})$.

The following conditions are equivalent for a point $U \in \operatorname{Sub}_{k}(\mathbb{V})$.

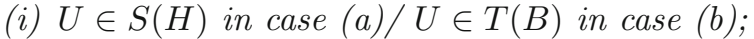

(ii) $U$ satisfies (*) with $\mathfrak{M}=\mathbf{P}_{k}(\mathbb{V})$;

(iii) $U$ satisfies (**) with $\mathfrak{M}=\mathbf{P}_{k}(\mathbb{V})$.

Then the following is immediate.

Lemma 3.5. Let $\Delta$ be a triangle in

(a) $\mathbf{P}_{k}^{\#}(\mathcal{L}(\xi))$ or in

(b) $\mathbf{P}_{k}(\mathcal{L}(\xi))$

Then one of the following holds:

(i) $\Delta$ is contained in $T_{\mathcal{L}}(B)$ for a $B \in S u b_{k+1}(\mathbb{V})$, where $\operatorname{ind}(\xi \uparrow B) \in$ $\{k, k+1\}$ in case $(a)$ and $\operatorname{ind}\left(\xi\lceil B)=k+1\right.$ in case $(b)$ (i.e. $\left.B \in \mathcal{L}(\xi)_{k+1}\right)$;

(ii) $\Delta$ is contained in $S_{\mathcal{L}(\xi)}(H)$ for an $H \in \mathcal{L}_{k-1}(\mathbb{V})$.

Let us fix $\mathcal{L}=\mathcal{L}(\xi)$. We start now with $\mathfrak{M}=\mathbf{P}_{k}^{\#}(\mathcal{L})$.

Proposition 3.6. Consider a triangle $\Delta$ in $\mathfrak{M}$ with the sides $q_{1}, q_{2}, q_{3}$ and the vertices $U_{1}, U_{2}, U_{3}$. Assume that

(a) $q_{1} \cup q_{2} \cup q_{3} \subset T_{\mathcal{L}}(B)$ for a $B \in S u b_{k+1}(\mathbb{V})$, or

(b) $q_{1} \cup q_{2} \cup q_{3} \subset S_{\mathcal{L}}(H)$ for an $H \in \mathcal{L}_{k-1}(\mathbb{V})$.

Then for every $U \in \mathcal{L}_{k}$ the following conditions are equivalent.

(i) $U \in T_{\mathcal{L}}(B)$ in case $(a) / U \in S_{\mathcal{L}}(H)$ in case (b);

(ii) (*) holds with $\mathfrak{M}=\mathbf{P}_{k}^{\#}(\mathcal{L})$;

(iii) (**) holds with $\mathfrak{M}=\mathbf{P}_{k}^{\#}(\mathcal{L})$. 
Proof. Implications (ii) $\vee($ iii $) \Longrightarrow$ (i) are evident in view of 3.4 .

To prove $(\mathrm{i}) \Longrightarrow(\mathrm{ii}) \wedge(\mathrm{iii})$ it suffices to use 3.2 and $3.3(\mathrm{ii})$ to see that corresponding variants of $(*)$ and $(* *)$ are true in $T_{\mathcal{L}}(B)$ and in $S_{\mathcal{L}}(H)$.

Lemma 3.7. Let $H \in \mathcal{L}_{k-1}, B \in \mathcal{L}_{k+1}$. Consider a triangle in $\mathbf{P}_{k}(\mathcal{L})$ with the sides $q_{1}, q_{2}, q_{3}$. Assume that

(a) $q_{1} \cup q_{2} \cup q_{3} \subset S_{\mathcal{L}}(H)$, or

(b) $q_{1} \cup q_{2} \cup q_{3} \subset T_{\mathcal{L}}(B)$.

The following conditions are equivalent for an $U \in \mathcal{L}_{k}$ :

(i) $U \in S_{\mathcal{L}}(H)$ in case $(a) / U \in T_{\mathcal{L}}(B)$ in case (b)

(ii) (**) holds with $\mathfrak{M}=\mathbf{P}_{k}(\mathcal{L})$.

Proof. As in the proof of 3.6 we observe that (ii) $\Longrightarrow$ (i) follows from 3.4.

In case (b) the implication (i) $\Longrightarrow$ (ii) is evident. In case (a) to justify (i) $\Longrightarrow$ (ii) we consider the plane $A$ in $S_{\mathcal{L}}(H)$ spanned in $\mathbf{P}_{k}(\mathcal{L})$ by $q_{1} \cup q_{2} \cup q_{3}$ and, analogously, the subspace $D$ spanned by $A \cup\{U\}$. Let us apply 3.2: $S_{\mathcal{L}}(H)$ restricted to $D$ is an exterior hyperbolic 3 -space (possibly degenerated and the claim follows by an elementary reasoning.

Note that, due to distinctions expressible in first order language between geometries induced on the elements of $\mathcal{S}_{k}(\mathcal{L}), \mathcal{T}_{k}(\mathcal{L})$, and $\mathcal{T}_{k}^{*}(\mathcal{L})$ (cf. 3.2 and 3.3) as a direct consequence of 3.6 and 3.7 we obtain

Corollary 3.8. The families $\mathcal{T}_{k}(\mathcal{L}), \mathcal{T}_{k}^{*}(\mathcal{L})$, and $\mathcal{S}_{k}(\mathcal{L})$ are definable in $\mathbf{P}_{k}^{\#}(\mathcal{L})$. The families $\mathcal{T}_{k}(\mathcal{L})$ and $\mathcal{S}_{k}(\mathcal{L})$ are definable in $\mathbf{P}_{k}(\mathcal{L})$.

Proof. Note, first, that projective geometry, affine geometry, and (generalized) hyperbolic geometry i.e. geometry of generalized hyperbolic spaces are elementarily distinguishable. A projective space is veblenian i.e. for any two its distinct lines $L_{1}, L_{2}$ the following holds:

if there are two other lines $L_{1}^{\prime}, L_{2}^{\prime}$ with a common point $a$ such that

$L_{1}, L_{2}$ both cross $L_{1}^{\prime}, L_{2}^{\prime}$ and both miss $a$ then $L_{1}$ crosses $L_{2}$.

In an affine space distinct lines which satisfy premises of (12) are disjoint iff they are parallel, and affine parallelism is a transitive relation. In both spaces $\mathbf{H}(\xi)$ and $\mathbf{H}^{*}(\xi)$ a 'parallelism' defined by the analogous requirement is not transitive.

Next, we remind that the space $\mathbf{H}(\xi)$ is a line space (each its two points are on a line) while $\mathbf{H}^{*}(\xi)$ is not linear.

In view of 3.2 and 3.3 to close the proof it suffices to distinguish $\mathcal{X}_{1}=T_{\mathcal{L}}(B)$ and $\mathcal{X}_{2}=S_{\mathcal{L}}(H)$ when restricted to $\mathbf{P}_{k}^{\#}(\mathcal{L})$ both carry geometry of a hyperbolic space. This is the case when $H \in \mathcal{L}_{k-1}, B \in \operatorname{Sub}_{k+1}(\mathbb{V})$, and $\xi\lceil B$ is 
nondegenerate of index $k$. In this case for every line $L$ of $\mathbf{P}_{k}^{\#}(\mathcal{L})$ contained in $\mathcal{X}_{i}$ we consider another extension of $L$ to star or top, resp. For $i=1$ every such a second extension is a star, which is a hyperbolic space. For $i=2$ there are in $\mathcal{X}_{i}$ lines extendable to a top being a projective space.

Note. By a side note let us observe that, generally, $\mathcal{X}_{1}$ and $\mathcal{X}_{2}$ as above are not isomorphic, unless they have the same dimension and index, which happens exactly when $n=2 k$ and $r=n-1$.

This completes our proof.

Note that the structures $\mathbf{H}(\xi)$ and $\mathbf{H}^{*}(\xi)$ are mutually definable for each nondegenerate $\xi$ (see 1.3). So, as a consequence of 3.8 and $3.2,3.3$ we conclude with

Proposition 3.9. The structures $\mathbf{P}_{k}(\mathcal{L})$ and $\mathbf{P}_{k}^{\#}(\mathcal{L})$ are mutually definable.

Proof. Indeed, to recover $\mathbf{P}_{k}(\mathcal{L})$ within $\mathbf{P}_{k}^{\#}(\mathcal{L})$ we note the following equivalence

let $q$ be a line of $\mathbf{P}_{k}^{\#}(\mathcal{L}) . q \in \mathcal{P}_{k}(\mathcal{L})$ iff $q \subset \mathcal{X}$ for an $\mathcal{X} \in \mathcal{T}_{k}(\mathcal{L})$.

To recover all the lines of $\mathbf{P}_{k}^{\#}(\mathcal{L})$ within $\mathbf{P}_{k}(\mathcal{L})$ let us follow the following steps. Let $q=[H, B]_{k} \cap \mathcal{L}_{k}$ be a line of $\mathbf{P}_{k}^{\#}(\mathcal{L})$. Consider $\mathcal{X}=S_{\mathcal{L}}(H) ; q \subset \mathcal{X}$ and the restriction of $\mathbf{P}_{k}(\mathcal{L})$ to $\mathcal{X}$ carries the geometry of $\left.\mathbf{H}^{*}\left(\xi \uparrow H^{\perp}\right)\right)$. In view of 1.3, the lines of $\mathbf{H}\left(\xi\left\lceil H^{\perp}\right)\right)$ - in particular, the line $q$ can be recovered in terms of the geometry induced on $\mathcal{X}$ by $\mathbf{P}_{k}(\mathcal{L})$.

Note that the map $\mathcal{S}_{k}(\mathcal{L}) \ni S_{\mathcal{L}}(H) \longmapsto H \in \mathcal{L}_{k-1}$ is a bijection.

Lemma 3.10. The family $\left\{\left\{S_{\mathcal{L}}(H): H \in q\right\}: q \in \mathcal{P}_{k-1}(\mathcal{L})\right\}$ is definable in $\mathbf{P}_{k}(\mathcal{L})$.

Proof. Let us fix a point $U$ on a line $[H, B]_{k} \subset T_{\mathcal{L}}(B) \in \mathcal{T}_{k}(\mathcal{L})$. Note that if $[E, B]_{k}$ with $E \in \mathcal{L}_{k-2}$ is a plane in $T_{\mathcal{L}}(B)$ then $[E, U]_{k-1}$ is a pencil $q$ in $\mathbf{P}_{k-1}(\mathcal{L})$. Clearly, $\left\{S_{\mathcal{L}}(H): H \in q\right\}=\left\{S \in \mathcal{S}_{k}(\mathcal{L}): U \in S, S \cap\right.$ $T_{\mathcal{L}}(B)$ is a line on $\left.[E, U]_{k}\right\}$.

So, we have justified our 'induction step':

Corollary 3.11. $\mathbf{P}_{k-1}(\mathcal{L})$ and $\mathbf{P}_{k-1}^{\#}(\mathcal{L})$ can be recovered within $\mathbf{P}_{k}(\mathcal{L})$ and within $\mathbf{P}_{k}^{\#}(\mathcal{L})$.

Finally, let us pay our attention to $\mathfrak{M}=\mathbf{G}_{k}(\mathcal{L})$. It is evident that the lines of $\mathbf{G}_{k}(\mathcal{L})$ are the elements of $\mathcal{T}_{k}(\mathcal{L})$. This yields immediately the following

Corollary 3.12. $\mathbf{G}_{k}(\mathcal{L})$ is definable in $\mathbf{P}_{k}^{\#}(\mathcal{L})$. 
In essence, we have a stronger result:

Proposition 3.13. The structures $\mathbf{P}_{k}(\mathcal{L})$ and $\mathbf{G}_{k}(\mathcal{L})$ are mutually definable.

Proof. Let us analyse the form of triangles in $\mathbf{G}_{k}(\mathcal{L})$ : let $\Delta$ be a such triangle and $U_{1}, U_{2}, U_{3}$ be its vertices. It is seen that then $H:=U_{1} \cap U_{2}=U_{1} \cap U_{3} \in$ $\mathcal{L}_{k-1}$. Consequently, $\Delta$ is a triangle in $\mathbf{P}_{k}(\mathcal{L})$. It is not too hard to observe that the analogue of $3.7\left(\right.$ a) with $\mathbf{P}_{k}(\mathcal{L})$ replaced by $\mathbf{G}_{k}(\mathcal{L})$ is valid. Consequently, the class $\mathcal{S}_{k}(\mathcal{L})$ is definable in $\mathbf{G}_{k}(\mathcal{L})$. Finally, we note that the class of lines of $\mathbf{P}_{k}(\mathcal{L})$ i.e. the class $\mathcal{P}_{k}(\mathcal{L})$ coincides with the class of non void intersections $\mathcal{X}_{1} \cap \mathcal{X}_{2}$, where $\mathcal{X}_{1}$ is a line of $\mathbf{G}_{k}(\mathcal{L})$ and $\mathcal{X}_{2} \in \mathcal{S}_{k}(\mathcal{L})$.

So, in view of 3.11 and 1.3, Propositions 3.9 and 3.13 prove 1.4 .

Open Access. This article is licensed under a Creative Commons Attribution 4.0 International License, which permits use, sharing, adaptation, distribution and reproduction in any medium or format, as long as you give appropriate credit to the original author(s) and the source, provide a link to the Creative Commons licence, and indicate if changes were made. The images or other third party material in this article are included in the article's Creative Commons licence, unless indicated otherwise in a credit line to the material. If material is not included in the article's Creative Commons licence and your intended use is not permitted by statutory regulation or exceeds the permitted use, you will need to obtain permission directly from the copyright holder. To view a copy of this licence, visit http://creativecommons. org/licenses/by/4.0/.

Publisher's Note Springer Nature remains neutral with regard to jurisdictional claims in published maps and institutional affiliations.

\section{References}

[1] Bachmann, F.: Aufbau der Geometrie aus dem Spiegelungsbegriff, 2nd edn Springer, Berlin (1973)

[2] Cameron, P.J.: Projective and polar spaces, QMW Math Notes 13. University of London, Queen Mary and Westfield College (1992)

[3] Cohen, A.: M Point-line spaces related to buildings. In: Buekenhout, F. (ed.) Handbook of incidence geometry, pp. 647-737. North-Holland, Amsterdam (1995)

[4] Pambuccian, V., Prażmowski, K., Sakowicz, K.: Defining co-punctuality in terms of line-orthogonality in plane hyperbolic geometry. Acta Math. Hungar. 109(4), 289-293 (2005)

[5] Pankov, M.: Grassmannians of classical buildings, Algebra and Discrete Mathematics, vol. 2. World Scientific, New Jersey (2010)

[6] Pankov, M., Prażmowski, K., Żynel, M.: Geometry of polar Grassmann spaces. Demonstr. Math. 39, 625-637 (2006)

[7] Prażmowska, M.: An axiom system describing degenerate hyperbolic planes in terms of directed parallelity. Demonstr. Math. 21(4), 913-941 (1988) 
[8] Prażmowska, M., Prażmowski, K.: Grassmann spaces over hyperbolic and quasihyperbolic spaces. Math. Pannonica 17(2), 195-220 (2006)

[9] Prażmowska, M., Prażmowski, K., Żynel, M.: Euclidean geometry of orthogonality of subspaces. Aequationes Math. 76(1-2), 151-167 (2008)

[10] Prażmowska, M., Prażmowski, K., Żynel, M.: Grassmann spaces of regular subspaces. J. Geom. 97, 99-123 (2010)

[11] Prażmowski, K.: An axiomatic description of the Strambach planes. Geom. Dedicata 32(2), 125-156 (1989)

[12] Radziszewski, K.: Subspaces and parallelity in semiaffine partial linear spaces. Abh. Math. Sem. Univ. Hamburg 73, 131-144 (2003)

K. Prażmowski

Department of Mathematics

University of Białystok

ul. Ciołkowskiego $1 \mathrm{M}$

15-245 Białystok

Poland

e-mail: krzypraz@math.uwb.edu.pl

Received: January 8, 2021.

Revised: May 27, 2021.

Accepted: May 28, 2021. 